\title{
Crumpling and buckling in membranes with hexagonal phase
}

\author{
ALDO PEZZUTTI, GASTON ARAUJO \\ Departament of Physics. Universidad Nacional del Sur \\ Bahía Blanca. ARGENTINA
}

\begin{abstract}
We study the dynamics of a copolymer membrane diblock in hexagonal phase. We call diblock copolymer membrane a copolymer film with thickness of the order of the size of the domains and that it presents the possibility of deforming freely. In a flat system of copolymer, the dynamics of coarsenig produces the relaxation of energy by diffusion and annihilation of defects. In a copolymer membrane, relaxation of the energy of the hexagonal phase resulting from a combined effect of the diffusion and annihilation of defects and of the buckling process.
\end{abstract}

Key-Words: - Buckling, Crystalline Membrane, Crumpling, Topological Defects.

Received: February 23, 2020. Revised: July 8, 2020. Accepted: July 15, 2020. Published: July 25, 2020.

\section{Introduction}

In recent years, there has been an increasing interest in the study of two-dimensional (2D) [1-6] textures on curved surfaces. This is motivated by the new properties that these systems present due to the coupling between the crystalline structure and the deformation metric. A clear example is observed in the dynamics of annihilation and buckling generated by the defect annihilation in graphene [7].

The study of crystalline arrangements of particles on curved surfaces has its origin more than 100 years ago with the Thompson problem [8]. The problem original refers to the ground state of a set of electrons, on a spherical shell. On the plane, the equilibrium configuration of a set of charged particles with the same sign is an arrangement or crystal lattice perfectly ordered hexagonal, that is to say free of defects.

The Gaussian curvature favors the formation of topological defects that they would be energetically prohibitive on flat crystals. The spherical shape has a positive Gaussian curvature that affects the equilibrium configuration of the particles confined in the shell. Balance settings contain defects on the hexagonal structure, something totally unexpected in a crystal flat. For spherical crystals with a low number of particles $(\sim 150)$, the settings balance equations contain 12 positive disclinations located in the vertices of an isocahedron [9].

The generalization of the Thompson problem includes biological systems, virus [10], colloidomas [11], pollen grains [12], systems of multielectrons in helium bubbles [13], fullerenes [14], etc.
In out-of-equilibrium systems, curvature also alters the dynamics of dislocations, through a geometric potential modifying the movement. Glide and Climb of dislocations [15].

The reverse problem is also very interesting. Suppose we have a system with crystalline order, what will happen if we allow the substrate to misshapen? When a defect is on a membrane with freedom displacement will produce an instability on the surface that deforms the surface. The problem is known by the name of buckling. The buckling process results in the shielding of the topological load of the defect by bending. Recently the buckling studio has awakened a high interest in graphene films [7]. Ripples on the membrane graphene alter the electronic properties of the material.

This study is focused on the description of the time evolution of a membrane with internal degrees of freedom that, below a critical temperature, undergoes a symmetry-breaking phase transition.

\section{Problem Formulation}

The dynamics of buckling on membranes has been studied using many different approaches, such as molecular dynamics [17] or Monte Carlo methods [18]. However, these approaches have a great computational complexity and the results are limited as regards simulation time and length scale of the represented system. The phase field model naturally incorporates the elasticity properties of the hexagonal phase and also provides an efficient approach over long periods of system evolution, in diffusive time scales. This approach has been used 
in order to describe a wide range of systems on both atomic and mesoscopic length scales. For instance, pattern formation [19], grain boundary melting [20], defect dynamics [21], symmetry-breaking phase transitions [22], block copolymers [23], Langmuir films [24], and liquid crystals [25].

To describe the dynamics of a crystalline membrane, we use a Brazovskii Hamiltonian model geometrically coupled to the topography of the membrane.

The evolution of the crystalline structure occurs by means of the dynamics dictated by the Brazovskii Hamiltonian model, which evolves coupled to the membrane deformation through the dynamics imposed by the Helfrich-Canham Hamiltonian model. In the following sections, we detail the elements that make up the proposed model.

\subsection{Fluid Membrane}

Fluid membranes occur in a wide variety of systems, from biological ones to condensed matter ones, such as surfactant films, vesicles, and lipid bilayer membranes [26-28].

During the last few years, the equilibrium phases and structures have been investigated intensively through different methods, including molecular dynamics [29], Monte Carlo [30] and phase-field models [31].

We employ a phase-field approach to describe the morphology of the membrane during a symmetrybreaking phase transition.

In the Monge gauge, the deformation of a membrane can be described by $\boldsymbol{r}(x, y)=$ $[\boldsymbol{x}, h(x, y)]$, where $\boldsymbol{x}=\{x, y\}$ represents a set of Cartesian coordinates in the plane, and $z=h(x, y)$ is the out-of-plane deformation over the reference plane $\boldsymbol{x}$ (see Fig. 1). The equilibrium properties of the fluid membrane are described by the Helfrich-Canham Hamiltonian model $F_{H C}$ [3236]:

$$
F_{H C}=\int\left[F_{1}+F_{2}+F_{3}\right] \sqrt{g} d x d y
$$

Here, $g$ is the metric tensor [35]. The first term $F_{1}=\frac{k_{B}}{2}\left(H_{m}-C_{0}\right)^{2}$ accounts for the bending elastic energy $[35,36]$. Where $k_{B}$ is the bending modulus, $H_{m}$ is the mean curvature, and $C_{0}$ is the homogeneous spontaneous curvature [37]. The second term $F_{2}=\sigma$ is an isotropic contribution; therefore, the membrane area is characterized by a surface tension $\sigma$. The third contribution $F_{3}=\frac{k_{g}}{2} R$ is a topological invariant that only depends on the genus of the surface. In this term, $k_{b}$ is the Gaussian rigidity and $R$ is the Gaussian curvature [38].

In the Monge gauge, the surface properties are defined as follows:

Tangent vectors to the surface:

$$
\begin{aligned}
& \overrightarrow{e_{1}}=\left(1,0, \frac{\partial h}{\partial x_{1}}\right)=\left(1,0, \partial_{1} h\right) \\
& \overrightarrow{e_{2}}=\left(0,1, \frac{\partial h}{\partial x_{2}}\right)=\left(0,1, \partial_{2} h\right)
\end{aligned}
$$

Normal vector:

$$
\vec{n}=\frac{\overrightarrow{e_{1}} \wedge \overrightarrow{e_{2}}}{\left|\overrightarrow{e_{1}} \wedge \overrightarrow{e_{2}}\right|}=\frac{1}{\sqrt{g}}\left(-\partial_{1} h,-\partial_{2} h, 1\right)
$$

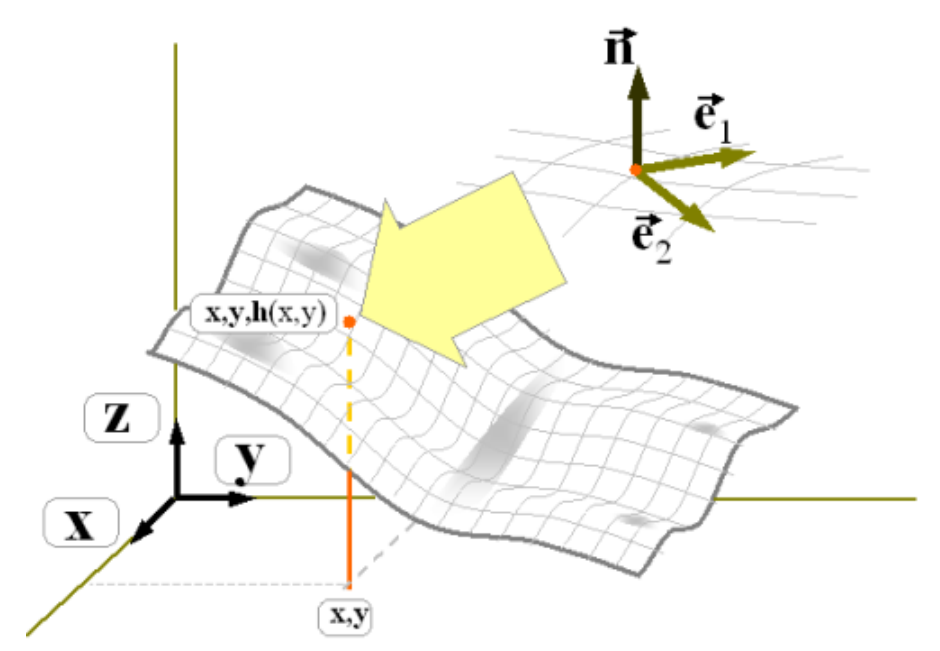

Figure 1. Monge gauge. The Monge gauge describes a surface by a single function $z=h(x, y)$, the height over a reference plane $(x, y)$.

This vectors follow the condition:

$$
\begin{gathered}
\overrightarrow{e_{l}} \cdot \overrightarrow{e_{j}}=\delta_{i, j} \\
\overrightarrow{e_{l}} \cdot \vec{n}=0
\end{gathered}
$$

Where $i, j=1,2$

The metric tensor $g_{i j}=\overrightarrow{e_{l}} \cdot \overrightarrow{e_{j}}$

$$
g_{i j}=\left(\begin{array}{ll}
\overrightarrow{e_{1}} \cdot \overrightarrow{e_{1}} & \overrightarrow{e_{1}} \cdot \overrightarrow{e_{2}} \\
\overrightarrow{e_{2}} \cdot \overrightarrow{e_{2}} & \overrightarrow{e_{2}} \cdot \overrightarrow{e_{1}}
\end{array}\right)
$$


In the Monge gauge is:

$$
g_{i j}=\left(\begin{array}{ll}
1+\left(\partial_{1} h\right)^{2} & \left(\partial_{1} h\right)\left(\partial_{2} h\right) \\
\left(\partial_{1} h\right)\left(\partial_{2} h\right) & 1+\left(\partial_{2} h\right)^{2}
\end{array}\right)
$$

And the inverse:

$$
g^{i j}=\frac{1}{g}\left(\begin{array}{cc}
1+\left(\partial_{2} h\right)^{2} & -\left(\partial_{1} h\right)\left(\partial_{2} h\right) \\
-\left(\partial_{1} h\right)\left(\partial_{2} h\right) & 1+\left(\partial_{1} h\right)^{2}
\end{array}\right)
$$

The determinant of the metric tensor in the Monge representation has the following expression:

$$
g=\left|g_{i j}\right|=\left(1+\left(\partial_{1} h\right)^{2}+\left(\partial_{2} h\right)^{2}\right)
$$

Two parameters characterize a surface, the mean curvature and the Gaussian curvature.

$$
H_{m}=\frac{1}{2}\left(\frac{1}{R_{1}}+\frac{1}{R_{2}}\right)
$$

Where are $R_{1}$ and $R_{2}$ the principals radius of curvature. is:

In the Monge parametrization, the mean curvature

$$
H_{m}=\frac{1}{2} K
$$

Where $K$ is the trace of curvature tensor $K_{i j}$

$$
K_{i j}=-\frac{1}{\sqrt{g}}\left(\begin{array}{ll}
\partial_{11} h & \partial_{12} h \\
\partial_{21} h & \partial_{22} h
\end{array}\right)
$$

The gaussian curvature:

$$
G=\frac{1}{R_{1}} \frac{1}{R_{2}}
$$

In the Monge parametrization, the gausssian curvature is:

$$
G=\frac{R}{2}=\frac{1}{g^{2}}\left(\partial_{11} h \partial_{22} h-\left(\partial_{12} h\right)^{2}\right)
$$

Whrere $R$ is the scalar curvature.

\subsection{Crystalline Phase}

The dynamics of buckling is studied through a continuous phase field model.

In the critical temperature surrounding, the orderdisorder transition is phenomenologically described by a Brazovskii Hamiltonian [29] modified to account for the geometry of the membrane:

$$
F_{\phi}=\int\left(2\left(\nabla_{L B}^{2}\right)^{2}-2 \nabla_{i} \phi \nabla^{i} \phi+\frac{\tau}{2} \phi^{2}+\frac{1}{4} \phi^{4}\right) d A
$$

Here we use the equation $\phi(\boldsymbol{r})=\psi(\boldsymbol{r})-\psi_{0}$, where $\psi(\boldsymbol{r})$ is the local composition, and $\psi_{0}$ is the average composition. The reduced temperature is represented by $\tau=\frac{T c_{-T}}{T_{C}}$ and the critical temperature by $T_{c}$. The expression of energy above is a modified version of the Ginzburg-Landau expansion. To incorporate the contribution of the surface geometry, the expression has been conveniently modified by using the differential operators. $\nabla_{L B}^{2}$ is the Laplace-Beltrami operator [36,37]. The standard convention of index summation has been used, where subscripts and superscripts indicate contravariant and covariant vectors, respectively. Below the critical temperature, the free-energy functional contributes to the formation of structures with hexagonal symmetry with a wavelength of $k_{0}=1 / \sqrt{2}[5]$.

\section{Crystalline Membrane Dynamics}

The total Hamiltonian that represents the dynamics of the membrane consists of the sum of the energies mentioned above, $F=F_{H C}+F_{\phi}$. A dissipative model [30] is used to obtain the time evolution of the membrane deformation, $h$ parameter, and composition, $\phi$. The dynamics of both order parameters is coupled through the membrane metric. The time evolution corresponds to the following equations:

$$
\begin{aligned}
& \frac{\partial \phi}{\partial t}=-\nabla_{L B}^{2}\left(\frac{\delta F}{\delta \phi}\right)+\eta_{\phi}(\boldsymbol{r}, t) \\
& \frac{\partial h}{\partial t}=-\left(\frac{\delta F}{\delta h}\right)+\eta_{h}(\boldsymbol{r}, t)
\end{aligned}
$$

Here, $\eta_{\phi}(\boldsymbol{r}, t)$ and $\eta_{h}(\boldsymbol{r}, t)$ are the random Gaussian noise [31].

Then the contribution of the Brazovskii energy in the evolution of the membrane shape $h$ intervenes through differential operations that appear in the Brazovskii energy and implicitly depend on $h$. 
The $\delta F / \delta \phi$ expression represents the variational derivative of free energy regarding the order parameter $\phi$, explicitly:

$$
\frac{\partial \phi}{\partial t}=\Delta \frac{\delta F}{\delta \phi}=\Delta\left[\Delta^{2} \phi+4 \Delta \phi+\tau \phi+\phi^{3}\right]
$$

The term $\delta F / \delta h$, represents the variation of the energy with respect to theparameter $h$. HelfrichCanham's energy related term incorporates explicitly its relation to the parameter $h$, however the energy of Brazovskii implicitly incorporates its relationship to deformation through differential operators.

Variation is calculated as follows, the variation of the position $\delta \vec{r}$ of any point that makes up the membrane correspond to a deformation $\psi$ in the normal direction $\delta \vec{r}=\chi \check{n}$. So, the

variational derivative $\delta F / \delta h$, can be rewritten in the function of $\psi$ of the following way:

$$
\left(\frac{\delta F}{\delta h}\right)=\left(\frac{\delta F}{\delta \chi}\right)
$$

the variation of energy is

$$
\delta F=\int\left[\left(F_{\phi}+F_{H C}\right) \delta d A+\delta\left(F_{\phi}+F_{H C}\right) d A\right]
$$

The complete expression is as follows:

$$
\begin{aligned}
& \frac{\partial h}{\partial t}=\left(F_{\phi}+F_{H C}\right) K+4 K^{i j} \nabla_{i} \phi \nabla_{j} \phi- \\
& \frac{4 K\left(\nabla_{L B}^{2} \phi\right)^{2}}{\sqrt{g}}+\quad K\left(\left(H_{m}-C_{0}\right)\left(R-K^{2}\right)-\right. \\
& \left.\nabla_{L B}^{2}\left(H-C_{0}\right)\right)
\end{aligned}
$$

\section{Numerical Simulation}

The equations (17) and (18) have been numerically solved using a semi-implicit pseudospectral algorithm with periodic boundary conditions. The time and spatial steps have been selected to provide numerical stability and precise resolution [39].

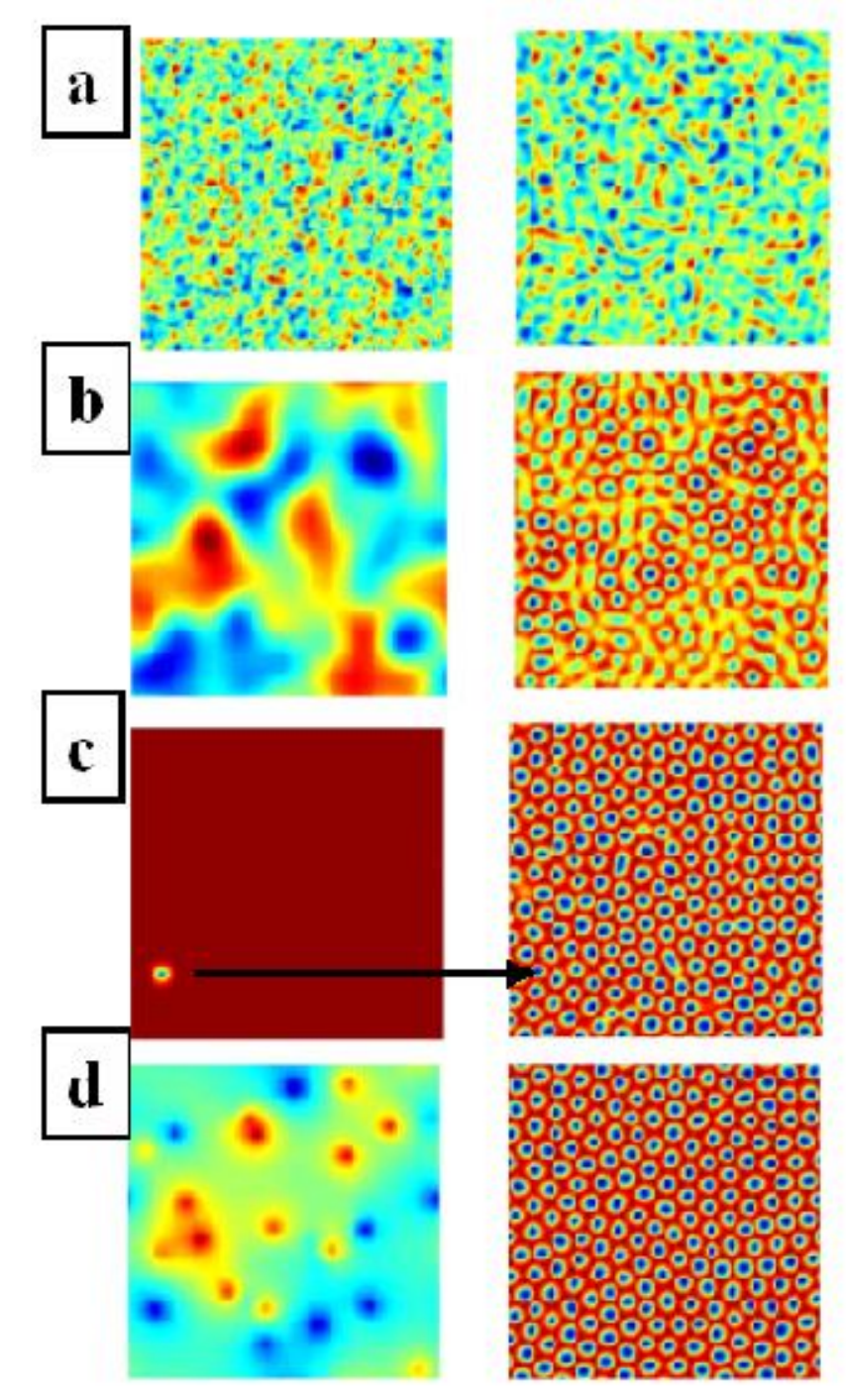

Figure 2. The images on the left correspond to the time evolution of the $h$ order parameter associated with the deformation height. The images on the right correspond to the time evolution of the order parameter $\phi$, i.e. the composition.

Figure $\mathbf{c}$ corresponds to the critical time $t_{c}$, where the coupling between deformation and composition that evolves in a coupled manner from that moment on begins to be observed.

Times: (a) $t=0$, (b) $t=50$ (c) $t=100$ (d) $t=10000$

The time step used is $\Delta t \sim 10^{-4}$, and the spatial discretization is $\Delta x \sim 10^{-2}$, both of which are typical values used in these numerical schemes. The size of the simulated system is $L x L=512 x 512$. In the appendix we detaild the code used.

Figure (2) illustrates the time evolution of both order parameters. The parameters used are the following: 
$\psi_{0}=0.4, \tau=2.1, \sigma=1.1, k_{B}=0.1$ and $\quad C_{0}=0$, according to the parameters presented in section 2.1 and 2.2, the values are chosen to represent a hexagonal membrane. The images on the left correspond to the time evolution of the $h$ order parameter associated with the deformation height. The images on the right correspond to the time evolution of the order parameter $\phi$, i.e. the composition. The figure show the coarsening kinetics of a crystalline membrane suffering a symmetry-breaking phase transition. A crystalline membrane can buckle out of the plane driven by the relaxation of the strain field introduced by topological defects. During the temporal evolution toward equilibrium the annihilation and diffusion of topological defects is dictated by the strong coupling between the membrane shape and the geometrically screened strain field associated with the defects.

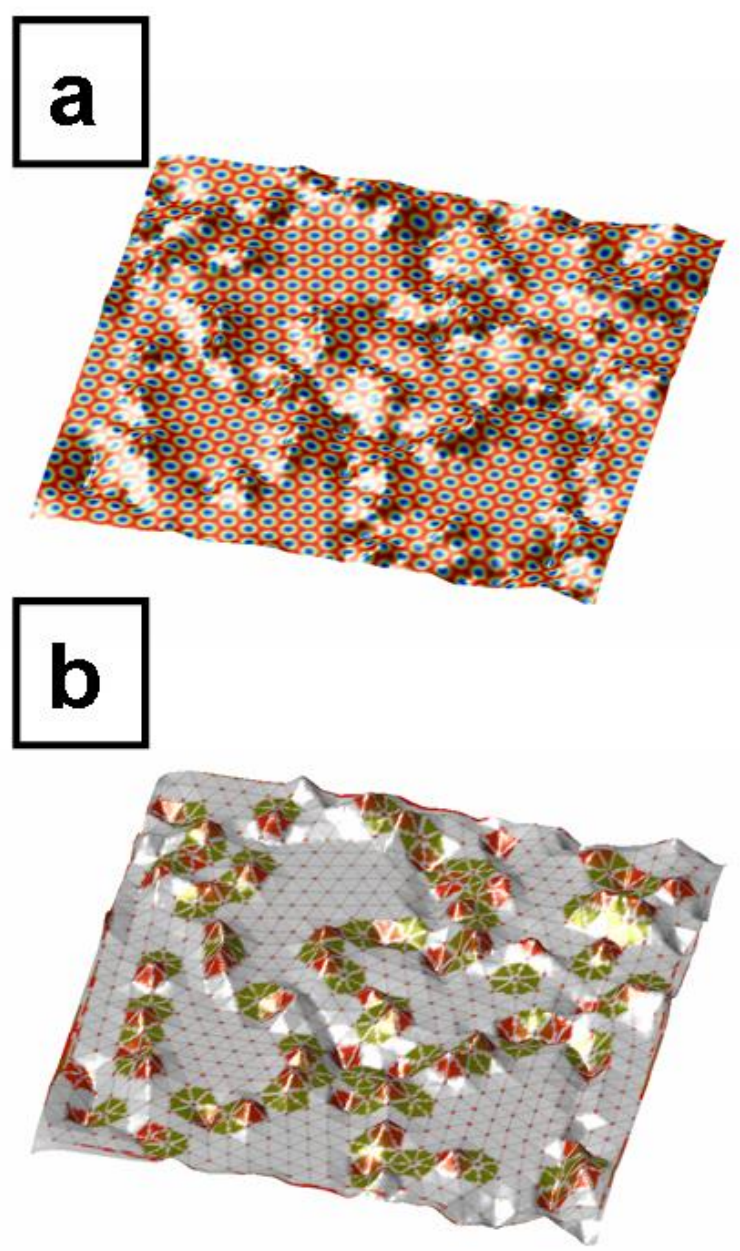

Figure 3. Hexagonal membrane. (a) Evolution of the membrane at long simulation times. (b) Identification of the topological defects of the hexagonal structure. Note how the deformation is strongly located on the grain boundaries.

\section{Buckling Dynamics}

At the mesoscopic level, the order within the hexagonal phase alters or modifies the stiffness constant of the membrane. As a result, the dynamics towards the equilibrium of the membrane is modified. A linear analysis of the bending energy predicts an exponential decay of the modes that constitute the initial condition of the membrane [40]. The amplitude of the different $q$ modes is obtained of the structure factor of the $h$ parameter. Note that we have used the $q$ notation to describe Fourier modes to avoid any confusion with the $k$ value of the bending stiffness. The structure factor can be expressed as:

$$
S_{h}(q)=\left\langle\left|h_{\vec{q}}\right|^{2}\right\rangle \sim \frac{1}{k q^{4}}
$$

Where $h_{\vec{q}}$ represents the Fourier Transform of $h$, and \langle\rangle represents the radial average over the wave vectors with equal $q$ modulus.

The dynamics of a membrane with crystalline structure is different from that of a liquid membrane [41], in which the $k$ stiffness is an isotropic variable. In membranes with crystalline order, the crystalline structure produces a modification in the typical dynamics of the q modes, presented in equation (5). In the literature, it has been shown that the stiffness of the membrane can be renormalized to write a membrane with crystalline order according to the expression [42]:

$$
\left\langle\left|h_{\vec{q}}\right|^{2}\right\rangle \sim q^{4-\eta}
$$

Where $\eta=2$, according to the theory of partially polymerized membranes [43]. Experimental work shows that the value changes with respect to the theoretical value between (2-3.1) for different degrees of polymerization [44]. The structure factor of the $h$ parameter allows the analysis of the dynamics of the membrane and the study of the influence of the crystalline structure on it.

\section{Results and Discussion}

The time evolution of the order parameter $\phi$ and the $h$ height are illustrated in Figure (2). When $\phi$ 
reaches a critical value, the coupling between both parameters $\phi$ and $h$ occurs (Image c, Figure 2).

The time when the coupling between both parameters occurs is identified as critical time $t_{c}$.

In this model, the elastic constants of the membrane are determined through the value of the order of composition parameter. For an hexagonal configuration, we get $\lambda=30\left|A_{k}\right|^{2} k_{0}^{2} \mu=6\left|A_{k}\right|^{2} k_{0}^{2}$ y $K_{0}=\frac{\mu(\mu+\lambda)}{2 \mu+\lambda}$ [45]. Where $\lambda, \mu$ are the Lamé constants and $K_{0}$ represents the Young modulus. In all cases, the parameters depend on the amplitude of the composition $A_{k}$, which results for an hexagonal configuration.

$A_{k}=\frac{1}{15}\left(-3 \psi_{0}+\right.$

$\left.\sqrt{3} \sqrt{20 k_{0}^{2}-20 k_{0}^{2}-5 \tau-12 \psi_{0}^{2}}\right)$ It is interesting to analyze the time evolution of the membrane deformation by calculating the structure factor of the $h$ parameter.

Figure (3) shows the hexagonal arrangement and the deformation produced by topological defects, Note how the deformation is strongly located on the grain boundaries.

This effect is also observed in Figure (4), where the temporal evolution is illustrated.

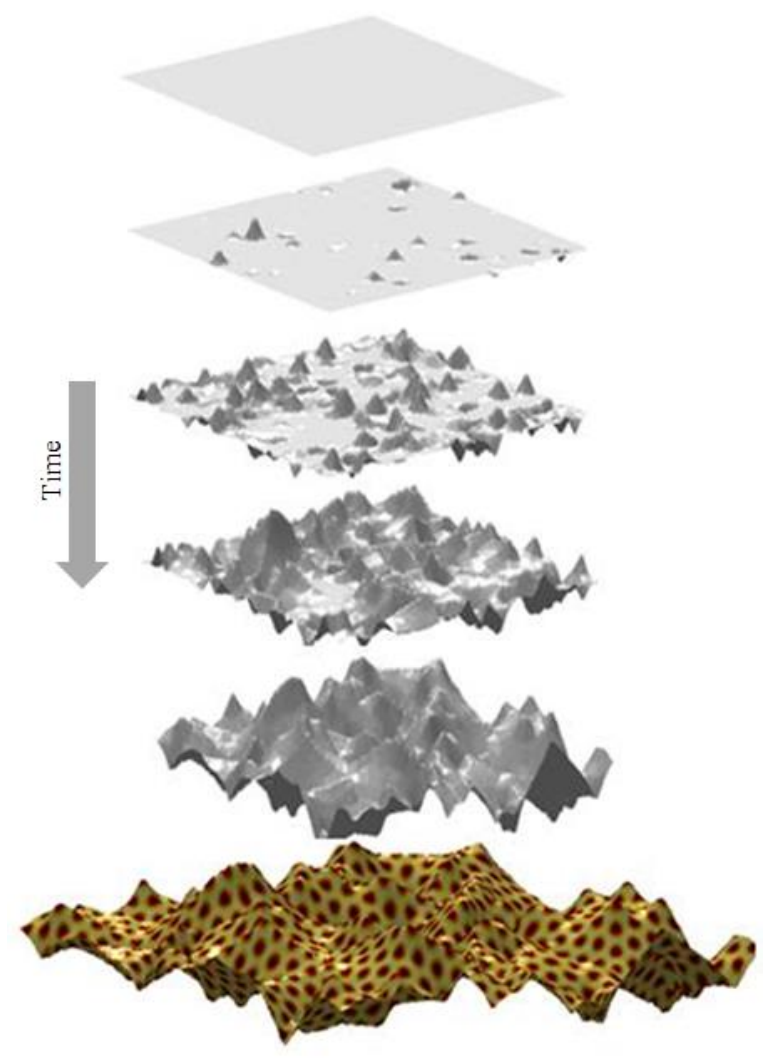

Figure. 4. Time evolution of the membrane. Only the hexagonal pattern is illustrated in the last figure

for clarity purposes and to better appreciate the evolution of the deformation, which corresponds to the time evolution shown in figure (2).

Figure (5) shows the structure factor for different simulation time values of an hexagonal-phase membrane. At short times, during the structure formation stage by spinodal decomposition, the dynamics between the parameters $\phi$ and $h$ is completely uncorrelated. In this time scale, we observe that the exponent $\left\langle\left|h_{\vec{q}}\right|^{2}\right\rangle \sim q^{-4.3 \pm 03}$ follows a similar law to the one predicted for a liquid membrane $\left\langle\left|h_{\vec{q}}\right|^{2}\right\rangle \sim q^{-4}$ [43].

When the value of the order parameter reaches a critical value, the coupling between the composition or crystal structure and the deformation of the membrane occurs. In this time, which we call critical time, it occurs in our simulation in $t_{c}=80$. Note in Figure (5) the change in the exponent at subsequent times to the critical time. The exponent value varies from $-4.3 \pm 0.3$ to $-1.8 \pm 0.2$. The change in the exponent characterizes the dynamics of the hexagonal-phase copolymer membrane. Note that the long time exponent is comparable to the value observed in polymer membranes with different degrees of polymerization [43].

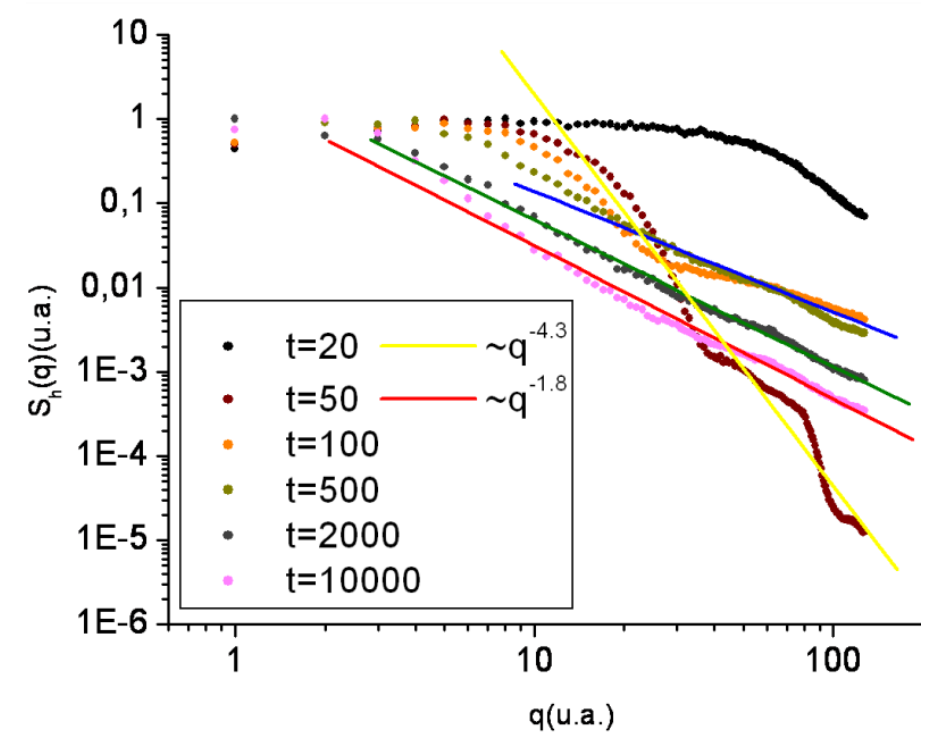

Figure 5. Structure factor of membrane deformation at different simulation times.

The structure deformation factor of the membrane for different simulation times is illustrated in the figure. Note the change of exponent in the power law of the structure factor with respect to the wave 
vector, which indicates a liquid membrane behavior at short times $\left(t<t_{c}\right)$ and a crystalline membrane one at long times $\left(t \gg t_{c}\right)$.

\section{Conclusion}

In summary, the evolution of an hexagonal membrane is analyzed by means of numerical simulation. The evolution towards the equilibrium of the order parameter that represents the hexagonal phase modifies the deformation dynamics of the membrane shape, which results in the variation of the elastic constants of the membrane.

The analysis by means of the structure factor of the evolution of the deformation of the membrane shows an evolution in early stages according to the dynamics of a liquid membrane and at long times the coupling with the hexagonal structure produces a dynamics according to the crystalline membrane. The coupling between the the topography of the membrane and the defects induces the pinning of positive disclinations and the formation of metastable structures of defects. The BrazovskiiHelfrich-Canham approach described here provides a general framework for studying the dynamics of topological defects in crystalline membranes, and can be straightforwardly extended to crystalline phases with different symmetries, in future works we will analyze the buckling dynamics of membranes with different internal structures.

\section{Appendix:}

The equations (17) and (18) have been numerically solved using a semi-implicit pseudospectral algorithm with periodic boundary conditions. The time and spatial steps have been selected to provide numerical stability and precise resolution see reference [39] for more details.

Numerical simulations are performed using the following code performed in the MATLAB language.

$\%$ numerical simulation to study the buckling process in a \%membrane with hexagonal order.

clear all

format long

tau $=0.3 \%$ reduce temperature

$\mathrm{U} 0=0.1 \%$ mean field

$\mathrm{Cf}=0 \%$ homogeneous spontaneous curvature $\mathrm{s}=0.33 \%$ surface tension del1 $=1 \%$ spatial step

del $2=1 \%$ spatial step

$\mathrm{nx}=256 \%$ matrix dimension

my $=256 \%$ matrix dimension

delt $=0.0005 \%$ temporal step

time $1=1500$

time $2=1000$

\%auxiliary matrix for the calculation of derivatives

for $\mathrm{n}=1: \mathrm{nx}$;

for $\mathrm{m}=1: \mathrm{my}$;

end

$\mathrm{A} 1(\mathrm{n}, \mathrm{m})=(\mathrm{i} / \mathrm{del} 1) *(\sin (2 * \mathrm{pi} *(\mathrm{n}-1) / \mathrm{nx}))$;

end

for $\mathrm{n}=1: \mathrm{nx}$;

for $\mathrm{m}=1: \mathrm{my}$

$\mathrm{A} 2(\mathrm{n}, \mathrm{m})=(\mathrm{i} / \mathrm{del} 2) *\left(\sin \left(2 * \mathrm{pi}^{*}(\mathrm{~m}-1) / \mathrm{my}\right)\right) ;$ end

end

for $\mathrm{n}=1: \mathrm{nx}$;

for $\mathrm{m}=1: \mathrm{my}$;

$\mathrm{A} 12(\mathrm{n}, \mathrm{m})=(-1 /(\operatorname{del} 1 * \operatorname{del} 2)) *(\sin (2 * \mathrm{pi} *(\mathrm{n}-$

1)/nx)* $\left.\sin \left(2 * \mathrm{pi}^{*}(\mathrm{~m}-1) / \mathrm{my}\right)\right)$;

end

end

for $\mathrm{n}=1: \mathrm{nx}$;

for $\mathrm{m}=1: \mathrm{my}$

$\mathrm{A} 11(\mathrm{n}, \mathrm{m})=\left(2 / \mathrm{del}^{\wedge} 2\right) *\left(\cos \left(2 * \mathrm{pi}^{*}(\mathrm{n}-1) / \mathrm{nx}\right)-\right.$

1);

end

end

for $\mathrm{n}=1: \mathrm{nx}$;

for $\mathrm{m}=1: \mathrm{my}$

$\mathrm{A} 22(\mathrm{n}, \mathrm{m})=(2 / \mathrm{del} 2 \wedge 2) *(\cos (2 * \mathrm{pi} *(\mathrm{~m}-1) / \mathrm{my})-$

1);

end

end

\%initial condition

$\mathrm{U}=(\operatorname{rand}(\mathrm{n}, \mathrm{m})-0.5) * 0.01+\mathrm{U} 0 ; \%$ order parameter

$\mathrm{h}=(\operatorname{rand}(\mathrm{n}, \mathrm{m})-0.5) * 0.001 ; \%$ deformation

\%time evolution

for $\mathrm{t}=1$ :time

for $\mathrm{t} 2=1$ :time 2

$\mathrm{hf}=\mathrm{fft} 2(\mathrm{~h})$;

$\mathrm{d} 1 \mathrm{~h}=\mathrm{real}\left(\mathrm{ifft} 2\left(\mathrm{~A} 1 *^{*} \mathrm{hf}\right)\right.$ );

$\mathrm{d} 2 \mathrm{~h}=\operatorname{real}($ ifft2(A2.*hf));

$\mathrm{d} 12 \mathrm{~h}=\operatorname{real}($ ifft2(A12.*hf)); 
$\mathrm{d} 21 \mathrm{~h}=\mathrm{d} 12 \mathrm{~h}$;

$\mathrm{d} 11 \mathrm{~h}=\mathrm{real}(\mathrm{ifft} 2(\mathrm{~A} 11 . * \mathrm{hf}))$;

$\mathrm{d} 22 \mathrm{~h}=\operatorname{real}\left(\mathrm{ifft} 2\left(\mathrm{~A} 22 .{ }^{*} \mathrm{hf}\right)\right)$;

$\%$ metric tensor components

$\mathrm{g}=\left(\right.$ ones $\left.(\mathrm{n}, \mathrm{m})+(\mathrm{d} 1 \mathrm{~h}) .^{\wedge} 2+(\mathrm{d} 2 \mathrm{~h}) .^{\wedge} 2\right)$;

$\mathrm{g} 11=\left(\right.$ ones $\left.(\mathrm{n}, \mathrm{m})+(\mathrm{d} 2 \mathrm{~h}) \cdot{ }^{\wedge} 2\right) \cdot / \mathrm{g}$;

g12 $=(-(\mathrm{d} 1 \mathrm{~h}) . *(\mathrm{~d} 2 \mathrm{~h})) \cdot / \mathrm{g}$

$\mathrm{g} 21=\mathrm{g} 12$;

$\mathrm{g} 22=\left(\right.$ ones $\left.(\mathrm{n}, \mathrm{m})+(\mathrm{d} 1 \mathrm{~h}) \cdot{ }^{\wedge} 2\right) . / \mathrm{g}$;

$\mathrm{R} 111=\mathrm{d} 1 \mathrm{~h} . * \mathrm{~d} 11 \mathrm{~h} . / \mathrm{g}$

$\mathrm{R} 112=\mathrm{d} 1 \mathrm{~h} . * \mathrm{~d} 12 \mathrm{~h} . / \mathrm{g}$

$\mathrm{R} 121=\mathrm{d} 1 \mathrm{~h} . * \mathrm{~d} 21 \mathrm{~h} . / \mathrm{g}$;

$\mathrm{R} 122=\mathrm{d} 1 \mathrm{~h} . * \mathrm{~d} 22 \mathrm{~h} . / \mathrm{g}$;

$\mathrm{R} 211=\mathrm{d} 2 \mathrm{~h} . * \mathrm{~d} 11 \mathrm{~h} . / \mathrm{g}$

$\mathrm{R} 212=\mathrm{d} 2 \mathrm{~h} . * \mathrm{~d} 12 \mathrm{~h} . / \mathrm{g}$;

$\mathrm{R} 221=\mathrm{d} 2 \mathrm{~h} . * \mathrm{~d} 21 \mathrm{~h} . / \mathrm{g}$;

$\mathrm{R} 222=\mathrm{d} 2 \mathrm{~h} . * \mathrm{~d} 22 \mathrm{~h} . / \mathrm{g}$;

$\mathrm{K} 11=-\mathrm{d} 11 \mathrm{~h} . /(\operatorname{sqrt}(\mathrm{g}))$;

$\mathrm{K} 12=-\mathrm{d} 12 \mathrm{~h} . /(\operatorname{sqrt}(\mathrm{g}))$;

$\mathrm{K} 21=\mathrm{K} 12$;

$\mathrm{K} 22=-\mathrm{d} 22 \mathrm{~h} . /(\operatorname{sqrt}(\mathrm{g}))$;

$\mathrm{KK} 11=\mathrm{g} 11 .{ }^{*}\left(\mathrm{~K} 11 .{ }^{*} \mathrm{~g} 11+\mathrm{K} 12 .{ }^{*} \mathrm{~g} 21\right)+\mathrm{g} 12 . *\left(\mathrm{~K} 21{ }^{*}\right.$ $\left.\mathrm{g} 11+\mathrm{K} 22 .{ }^{*} \mathrm{~g} 21\right)$;

$\mathrm{KK} 12=\mathrm{g} 11 . *\left(\mathrm{~K} 11 .{ }^{\mathrm{g}} 12+\mathrm{K} 12 .{ }^{*} \mathrm{~g} 22\right)+\mathrm{g} 12 . *\left(\mathrm{~K} 21{ }^{*}\right.$ $\left.\mathrm{g} 12+\mathrm{K} 22 .{ }^{*} \mathrm{~g} 22\right)$;

$\mathrm{KK} 21=\mathrm{g} 21 . *\left(\mathrm{~K} 11 .{ }^{*} \mathrm{~g} 11+\mathrm{K} 12 .{ }^{*} \mathrm{~g} 21\right)+\mathrm{g} 22 . *\left(\mathrm{~K} 21{ }^{*}\right.$ $\mathrm{g} 11+\mathrm{K} 22 . * \mathrm{~g} 21)$;

$\mathrm{KK} 22=\mathrm{g} 21 . *\left(\mathrm{~K} 11 .{ }^{*} \mathrm{~g} 12+\mathrm{K} 12 .{ }^{*} \mathrm{~g} 22\right)+\mathrm{g} 22 . *(\mathrm{~K} 21 . *$ $\mathrm{g} 12+\mathrm{K} 22 . * \mathrm{~g} 22)$;

$\mathrm{K}=-(\mathrm{d} 11 \mathrm{~h}+\mathrm{d} 22 \mathrm{~h}) \cdot /(\operatorname{sqrt}(\mathrm{g}))+\left(\left((\mathrm{d} 1 \mathrm{~h}) \cdot{ }^{\wedge} 2\right) \cdot{ }^{*} \mathrm{~d} 11 \mathrm{~h}+\right.$ $2 * d 1 h . * d 2 h . * d 12 h+((\mathrm{d} 2 \mathrm{~h}) . \wedge 2) . * d 22 \mathrm{~h}) . / \operatorname{sqrt}\left(\mathrm{g} .{ }^{\wedge} 3\right) ;$

$\mathrm{R}=2 *(\mathrm{~d} 11 \mathrm{~h} . * \mathrm{~d} 22 \mathrm{~h}-(\mathrm{d} 12 \mathrm{~h}) . \wedge 2) . / \mathrm{g} .{ }^{\wedge} 2$;

$\mathrm{UF}=\mathrm{fft} 2(\mathrm{U})$;

$\mathrm{d} 1 \mathrm{U}=\mathrm{real}\left(\mathrm{ifft} 2\left(\mathrm{~A} 1{ }^{*} \mathrm{UF}\right)\right.$ );

$\mathrm{d} 2 \mathrm{U}=\mathrm{real}\left(\mathrm{ifft} 2\left(\mathrm{~A} 2{ }^{*} \mathrm{UF}\right)\right.$ );

$\mathrm{d} 12 \mathrm{U}=\mathrm{real}\left(\mathrm{ifft} 2\left(\mathrm{~A} 12 .{ }^{*} \mathrm{UF}\right)\right.$ );

$\mathrm{d} 21 \mathrm{U}=\mathrm{d} 12 \mathrm{U}$;

d11U $=$ real(ifft2(A11.*UF));

$\mathrm{d} 22 \mathrm{U}=\mathrm{real}\left(\mathrm{ifft} 2\left(\mathrm{~A} 22 .{ }^{*} \mathrm{UF}\right)\right.$ );

$\% \% \% \%$ operador laplace-beltrami

$\mathrm{LAU}=\mathrm{g} 11 . * \mathrm{~d} 11 \mathrm{U}+2 * \mathrm{~g} 12 . * \mathrm{~d} 12 \mathrm{U}+\mathrm{g} 22 . * \mathrm{~d} 22 \mathrm{U}-$ $\left(\mathrm{g} 11 . * \mathrm{R} 111+2 * \mathrm{~g} 12 . * \mathrm{R} 112+\mathrm{g} 22 .{ }^{*} \mathrm{R} 122\right) . * \mathrm{~d} 1 \mathrm{U} \quad-$ (g11.*R211+2*g12.*R212+g22.*R222).*d2U;
GRADU $=\left((1+(\mathrm{d} 2 \mathrm{~h}) . \wedge 2) \cdot *(\mathrm{~d} 1 \mathrm{U}) \cdot{ }^{\wedge} 2\right.$

$2 *$ d 1 h. $*$ d2h.*d1U.*d2U

$(1+(\mathrm{d} 1 \mathrm{~h}) . \wedge 2) . *(\mathrm{~d} 2 \mathrm{U}) . \wedge 2) \cdot / \mathrm{g}$

$\mathrm{UK}=\mathrm{fft} 2(\mathrm{~K}-\mathrm{Cf})$;

$\mathrm{d} 1 \mathrm{~K}=\mathrm{real}(\mathrm{ifft} 2(\mathrm{~A} 1 . * \mathrm{UK}))$;

$\mathrm{d} 2 \mathrm{~K}=\mathrm{real}(\mathrm{ifft} 2(\mathrm{~A} 2 . * \mathrm{UK}))$;

$\mathrm{d} 12 \mathrm{~K}=\operatorname{real}(\mathrm{ifft} 2(\mathrm{~A} 12 . * \mathrm{UK}))$;

$\mathrm{d} 21 \mathrm{~K}=\mathrm{d} 12 \mathrm{~K}$;

$\mathrm{d} 11 \mathrm{~K}=\mathrm{real}(\mathrm{ifft} 2(\mathrm{~A} 11 . * \mathrm{UK}))$;

$\mathrm{d} 22 \mathrm{~K}=\operatorname{real}\left(\mathrm{ifft} 2\left(\mathrm{~A} 22 .{ }^{*} \mathrm{UK}\right)\right)$;

$\mathrm{LAK}=\mathrm{g} 11 . * \mathrm{~d} 11 \mathrm{~K}+2 * \mathrm{~g} 12 . * \mathrm{~d} 12 \mathrm{~K}+\mathrm{g} 22 . * \mathrm{~d} 22 \mathrm{~K}-$ (g11.*R111+2*g12.*R112+g22.*R122).*d1K (g11.*R211+2*g12.*R212+g22.*R222).*d2K;

$\mathrm{H} 1=\mathrm{A} *\left((\mathrm{~K}-\mathrm{Cf}) \cdot *\left(\mathrm{R}-\mathrm{K} .{ }^{\wedge} 2\right)-\mathrm{LAK}\right)$;

$\mathrm{H} 2=4 *(\mathrm{KK} 11 . * \mathrm{~d} 1 \mathrm{U} . * \mathrm{~d} 1 \mathrm{U}+\mathrm{KK} 12 . * \mathrm{~d} 1 \mathrm{U} . * \mathrm{~d} 2 \mathrm{U}+$ $\mathrm{KK} 21 . * \mathrm{~d} 2 \mathrm{U} . * \mathrm{~d} 1 \mathrm{U}+\mathrm{KK} 22 . * \mathrm{~d} 2 \mathrm{U} . * \mathrm{~d} 2 \mathrm{U}) ;$

$\mathrm{H} 3=-4 *(\mathrm{~K} . *(\mathrm{LAU}) . \wedge 2) . / \operatorname{sqrt}(\mathrm{g})$;

$\mathrm{H}=\left((-2 * \mathrm{GRADU}+2 *(\mathrm{LAU}))^{\wedge} 2+(\mathrm{tau} / 2) * \mathrm{U} \cdot \cdot^{\wedge} 2+\right.$ $\left.\left.(1 / 4)^{*} \mathrm{U} . \wedge 4\right)+(\mathrm{A} / 2)^{*}(\mathrm{~K}-\mathrm{Cf}) . \wedge 2+\mathrm{s}\right)$

$\mathrm{T}=-(\mathrm{H} . * \mathrm{~K}+\mathrm{H} 1+\mathrm{H} 2+\mathrm{H} 3)$

\%laplace beltrami operator

$\mathrm{UF} 2=\mathrm{fft} 2(\mathrm{LAU})$

d1U $=$ real(ifft2(A1.*UF2));

$\mathrm{d} 2 \mathrm{U}=\mathrm{real}\left(\mathrm{ifft} 2\left(\mathrm{~A} 2{ }^{*} \mathrm{UF} 2\right)\right.$ )

$\mathrm{d} 12 \mathrm{U}=\mathrm{real}(\mathrm{ifft} 2(\mathrm{~A} 12 . * \mathrm{UF} 2))$;

$\mathrm{d} 21 \mathrm{U}=\mathrm{d} 12 \mathrm{U}$;

$\mathrm{d} 11 \mathrm{U}=\mathrm{real}(\mathrm{ifft} 2(\mathrm{~A} 11 . * \mathrm{UF} 2))$;

d22U $=$ real(ifft2(A22.*UF2));

$\mathrm{LAU} 1=\mathrm{g} 11 . * \mathrm{~d} 11 \mathrm{U}+2 * \mathrm{~g} 12 . * \mathrm{~d} 12 \mathrm{U}+\mathrm{g} 22 . * \mathrm{~d} 22 \mathrm{U}$ $-(\mathrm{g} 11 . * \mathrm{R} 111+2 * \mathrm{~g} 12 . * \mathrm{R} 112+\mathrm{g} 22 . * \mathrm{R} 122) \cdot{ }^{*} \mathrm{~d} 1 \mathrm{U} \quad-$ (g11.*R211+2*g12.*R212+g22.*R222).*d2U;

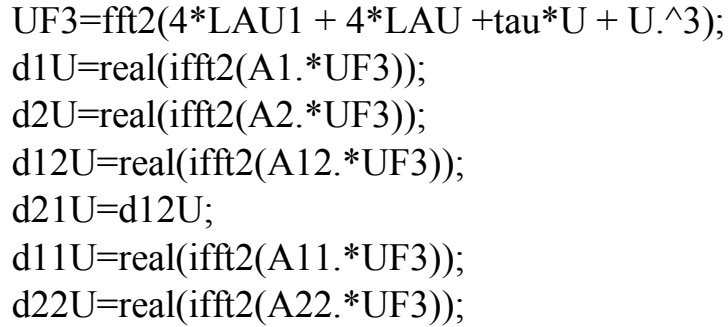

LAU2 $=\mathrm{g} 11 . * \mathrm{~d} 11 \mathrm{U}+2 * \mathrm{~g} 12 . * \mathrm{~d} 12 \mathrm{U}+\mathrm{g} 22 . * \mathrm{~d} 22 \mathrm{U}$ $-(\mathrm{g} 11 . * \mathrm{R} 111+2 * \mathrm{~g} 12 . * \mathrm{R} 112+\mathrm{g} 22 . * \mathrm{R} 122) * \mathrm{~d} 1 \mathrm{U} \quad-$ $\left(\mathrm{g} 11 . * \mathrm{R} 211+2 * \mathrm{~g} 12 .{ }^{*} \mathrm{R} 212+\mathrm{g} 22 .{ }^{*} \mathrm{R} 222\right) .{ }^{*} \mathrm{~d} 2 \mathrm{U}$; 
$\% \% \% \% \% \% \%$

$\mathrm{h}=$ delt $* \operatorname{sqrt}(\mathrm{g}) . * \mathrm{~T}+\mathrm{h}$;

$\mathrm{U}=\mathrm{U}+$ delt $* \mathrm{LAU} 2$;

end

$\% \% \%$ order parameter and deformation

pcolor(real(U)), shading interp, ...

axis('off'), axis('equal'), title(t);

figure

pcolor(real(h)), shading interp, ...

axis('off'), axis('equal'), title(t);

\section{References:}

[1]Duclos, G., Adkins, R., Banerjee, D., Peterson, M. S., Varghese, M., Kolvin, I., Toschi, F. Science, 367 (2020). 6482.

[2]Koning, V., Vitelli, V. Crystals and liquid crystals confined to curved geometries. Fluids, Colloids and Soft Materials: An Introduction to Soft Matter Physics, 369-386. (2016).

[3] Chantawansri T. L., Bosse A. W., Hexemer A., Ceniceros A. D. C., Garcia-Cervera J., Kramer E. J. and. Fredrickson, G. H Phys. Rev. E 75 (2007) 031802.

[4] GiomiL., Bowick M., Phys. Rev. B 76 (2007) 054106

[5] Kim, S. C., Yu, L., Pertsinidis, A., Ling, X. S. Proceedings of the National Academy of Sciences (2020).

[6] Alexandre A. Mazaleyrat, E., Förster, G. D., Tonnoir, C., Gilles, B., David, P. 2 D Materials 7 (2020) 025016.

[7] J. J. Thomson. Philos. Mag., 7 (1904) 237.

[8] T. Kohyama, D. M. Kroll, and G. Gompper. Phys. Rev. E, 68 (2003) 061905.

[9] V. Y. Klishko J. T. Finch B. K. Ganser, Su Li and W. I. Sundquist. Science, 283 (1999) 80.

[10] A. Cacciuto A. D. Dinsmore M. F. Hsu D. R. Nelson M. G. Nikolaides A. Travesset A. R. Bausch1, M. J. Bowick and D. A.Weitz.. Science, 299 (2003) 1716.

[11] S. C. O0Brien R. F. Curl H. W. Kroto, J. R. Heath and R. E. Smalley Nature, 318 (1985)162.

[12] P. Leiderer, Z. Phys. B, 98 (1995) 303.

[13] J. B. Lucks V. Vitelli and D. R. Nelson. PNAS, 103 (2006) 12322.

[14] J. M. Carlsson. Nature, 6 (2007) 801.
[15] Cavallo F., Lagally M. G., Soft Matter 6 (2010)439.

[16] Harden J. L., MacKintosh F. C., Olmsted P. D., Phys. Rev. E 72 (2005) 011903.

[17] Lowengrub J. S., Raätz A., Voigt A., Phys. Rev. E 72 (2009) 011903.

[18] Moldovan D., Golubonic L., Phys. Rev. E 60 (1999) 4377.

[19] Popova H., Milchev A., Phys. Rev. E, 77 (2008) 041906.

[20] Park J. M., Lubensky T. C., Phys. Rev. E 53, (1996) 2648.

[21] Park J. M., Lubensky T. C., J. Phys. I (France) 6, (1996) 493.

[22] Solis, F. J. Funkhouser C. M., Thornton K., Europhys. Lett. 82 (2008) 38001.

[23] Canham P. B., J. Theor Biol. 26 (1970) 61.

[24] Helfrich Z., Naturforsch C 28 (1973) 693.

[25] Funkhouser C. M., Solis F. J., Thornton K., Phys Rev. E 76 (2007) 011912.

[26] Sokolnokoff I. S., Theory and Applications to Geometry and Mechanics of Continua (Wiley, New York, 1964).

[27] Seul M., Andelman D., Science 267 (1997) 476.

[28] Mellenthin J., Karma A., Plapp M., Phys. Rev. B 78 (2008) 184110.

[29] Elder K. R., Katakowski M., Haataja M., Grant M., Phy Rev. Lett. 88 (2002) 245701.

[30] Yamada K., Komura S., J. Phys. Condens. Matter 20 (2008) 1.

[31] Hamley I. W., The Physics of Block Copolymers (Oxford University Press, New York 1998).

[32] Andelman A., Brochard F., Joanny J., $J$. Chem. Phys. 86 (1987) 3673.

[33] Swift J., Phys. Rev. A 14 (1976) 2274.

[34] Brazovskii S. A., Sov. Phys. JETP 41 (1975) 85.

[35] Nelson D. R., Defects and Geometry in Condensed Matter Physics (Cambridge University Press, Cambridge United Kingdom, 2002).

[36] Kamien R. D. Rev. Mod. Phys. 74 (2002) 953.

[37] Yang O., Zhong C., Helfrich W. Phys Rev A 39 (1989) 5280.

[38] Chaikin P. M., Lubensky T. C., Principles of Condensed Matter Physics (Cambridge University Press, Cambridge, United Kingdom, 1995). 
[39] Funkhouser C. M., Solis F. J., Thornton K., Phys Rev. E 76 (2007) 011912.

[40] Vellab D., Adda-Bediab M., Davidovich B., Schrolla R. D., Cerda E. A., PNAS 108 (2011) 18227.

[41] Chaieb S., Natrajan V. K., El-Rahman a. A., Phys. Rev, Lett. 96 (2006) 078101.

[42] Nelson D. R., Radzibovsky L., Phys. Rev. A 46 (1992) 7474.

[43] Kawasaki K. Otha T., Physica A 139 (1986) 223.

[44] de Jeu W. H., Cerda E., Emrick T., Menon N., Huang J., Jusz-kiewicz M., Russell T. P., Science 317 (2007) 650.

\section{Contribution of individual authors to the creation of a scientific article (ghostwriting policy)}

Aldo Pezzutti and Gastón Araujo have contributed equally in the writing of the article and in the simulation and analysis of data.

\section{Sources of funding for research} presented in a scientific article or scientific article itself

This work was supported by Universidad N acional del Sur, and the National Research Council of Argentina (CONICET).

\section{Creative Commons Attribution}

\section{License 4.0 (Attribution 4.0}

International, CC BY 4.0)

This article is published under the terms of the Creative Commons Attribution License 4.0

https://creativecommons.org/licenses/by/4.0/deed.en US 